\title{
Subtyping Salmonella isolated from pet dogs with multilocus sequence typing (MLST) and clustered regularly interspaced short palindromic repeats (CRISPRs)
}

\author{
Cheng Yang ${ }^{1,2}$, Wangfeng Shao ${ }^{1,2}$, Lingling Wei ${ }^{1}$, Lingxiao Chen ${ }^{1}$, Aihua Zhu ${ }^{1 *}\left(\mathbb{D}\right.$ and Zhiming Pan ${ }^{3,4^{*}}$
}

\begin{abstract}
Salmonella, as a zoonotic pathogen, has attracted widespread attention worldwide, especially in the transmission between household pets and humans. Therefore, we investigated the epidemic distribution of dog Salmonella from pet hospitals and breeding base in Xuzhou, Jiangsu Province, China, and used multilocus sequence typing (MLST) and clustered regularly interspaced short palindromic repeats (CRISPRs) to subtype Salmonella isolates. From April 2018 to November 2019, a total of 469 samples were collected from pet hospitals and breeding base, including 339 dog samples and 60 cat samples. S. Kentucky (40.74\%) was the most prevalent serotype, but other, such as S. Typhimurium (18.52\%) and S. Indiana (18.52\%), were also widespread. Eight different sequence type (ST) patterns were identified by MLST and ST198 was the highest proportion of these isolates. CRISPRs analysis showed that 9 different Kentucky CRISPR types (KCTs) was identified from ST198. 48 spacers including 29 (6 News) for CRISPR1 and 19 (4 News) for CRISPR2 that proved the polymorphic of Salmonella genes in samples from different sources. The analysis demonstrated that the common serotypes were widely present in pet hosts in the same area. This analysis shows that CRISPR genes have better recognition ability in the same serotype, which has a positive effect on the traceability of Salmonella and the prevention and treatment of salmonellosis.
\end{abstract}

Keywords: Salmonella, Pet, Multilocus sequence typing, Clustered regularly interspaced short palindromic repeats, Molecular subtyping

\section{Introduction}

Salmonella, as a kind of zoonotic bacteria, has caused widespread concern worldwide. In recent years, the number of pets worldwide has increased dramatically, of which cats and dogs account for $80 \%$. The close contact between humans and pets also greatly increases the risk of Salmonella spreading between pets and humans.

\footnotetext{
*Correspondence: ahzhu@jsnu.edu.cn; pzmshq@163.com

${ }^{1}$ College of Health Sciences, Jiangsu Normal University, Xuzhou 221116, Jiangsu, China

${ }^{3}$ Jiangsu Co-Innovationnovation Center for Prevention and Control of Important Animal Infectious Diseases and Zoonoses, Yangzhou University, Yangzhou 225009, Jiangsu, China

Full list of author information is available at the end of the article
}

Previous investigations showed that the positive rate of Salmonella in pet dogs with diarrhea is more than twice that of healthy pet dogs (Renate Reimschuessel et al. 2017). To make matters worse, healthy pets with no obvious symptoms will also release Salmonella to the outside environment in the form of feces, causing cross-infection between humans and other animals (Wray and Wray 2000). Salmonella can also spread among animal groups (Van Immerseel et al. 2004). There are even individual cases that can prove that pet dogs and cats can spread their own Salmonella to humans (Cherry et al. 2004; Koehler et al. 2006). From 2006 to 2008, in the United States, a study related to human Salmonella infection and contaminated dry dog and cat food showed that Salmonella 
is more likely to spread between infants and young children (Behravesh et al. 2010). Interestingly, In southern Ontario, Canada, Murphy tested 188 dogs and 39 cats for Salmonella, and found no positive strains (Murphy et al. 2009). Therefore, it is necessary to pay more attention to Salmonella from pets and Salmonellosis prevention in China which have the largest number of pets.

The genetic characteristics of pet-derived Salmonella have not been extensively studied. At present, molecular detection methods are widely used in the typing of Salmonella strains. Especially MLST, which is more efficient and accurate than pulse field gel electrophoresis (PFGE), and is more suitable for the identification and differentiation of animal Salmonella (Shi et al. 2013). The results of MLST are helpful to analyze the genetic characteristics of different serotypes of Salmonella, and prove the existence of microevolution between different STs. On the other hand, for the same serotype, MLST could not accurately demonstrate the genetic and phenotypic differences of Salmonella (Tennant et al. 2010; Gymoese et al. 2017). Therefore, a new identification method was established (Fabre et al. 2012). CRISPR has a higher resolution for identical serotypes than PFGE (Thompson et al. 2018). This study investigates the genetic diversity and subtype transmission of pet Salmonella between pets and humans in Xuzhou, China. Two molecular typing methods were used. 27 strains of Salmonella from pets were identified with different subtypes using MLST; CRISPR was used to analyze the identical serotypes of $S$. Kentucky and estimate the potential risk of the same subtypes Salmonella spreading between pets and humans.

\section{Materials and methods}

\section{Sample collection and Serotyping}

From April 2018 to November 2019, 469 samples were from 8 different pet hospitals and a breeding base, of which 339 were from dogs and 130 were from cats. Rectal sampling of pets using cotton swabs infiltrated with BPW and then strictly followed the Chinese National Standard Method (GB 4789.4-2016) for Salmonella isolation, the serotypes of all of the strains determined by slide agglutination according to the Kauffman-White serotyping scheme with $\mathrm{O}$ - and $\mathrm{H}$-antigen specific sera.

\section{Multilocus sequence typing (MLST)}

The confirmed strains were cultured with LB medium at $37^{\circ} \mathrm{C}$ and $120 \mathrm{rpm} / \mathrm{min}$ shaking for $18 \mathrm{~h}$, Then genomic DNA was extracted with the TIANamp Bacteria DNA kit (Tiangen Biotech (Beijing) CO., LTD, China) according to the manufacturer's protocol. Each identified strain was characterized by MLST using seven housekeeping (Table 1) (Kidgell et al. 2002). PCR products were delivered to Sangon Biotech (Shanghai) Co., Ltd. for purification and sequencing, and the seven pairs of housekeeping gene testing and sequencing results of each strain were uploaded to the MLST database for comparison (http:// mlst.warwick.ac.uk/mlst/dbs/ Senterica). This is used to determine the sequence type (ST) of each strain. A minimum spanning tree was generated using BioNumerics software, version 7.5 (Applied Maths, Kortrijk, Belgium) to analyze the distribution of STs from cats, dogs and humans.

Table 1 Sequencing and amplification primers for Salmonella MLST typing

\begin{tabular}{|c|c|c|c|}
\hline \multirow[t]{2}{*}{ Gene } & \multicolumn{2}{|l|}{ Primers } & \multirow[t]{2}{*}{ Size(bp) } \\
\hline & Amplification primers $\left(5^{\prime}-3^{\prime}\right)$ & Sequencing primers $\left(5^{\prime}-3^{\prime}\right)$ & \\
\hline \multirow[t]{2}{*}{ aroc } & F: CCTGGCACCTCGCGCTATAC & F: GGCGTGACGACCGGCAC & 826 \\
\hline & R: CCACACACGGATCGTGGCG & R: AGCGCCATATGCGCCAC & \\
\hline \multirow[t]{2}{*}{ dnaN } & F: ATGAAATTTACCGTTGAACGTGA & F: CCGATTCTCGGTAACCTGCT & 833 \\
\hline & R: AATTTCTCATTCGAGAGGATTGC & R: ACGCGACGGTAATCCGGG & \\
\hline \multirow[t]{2}{*}{ hemD } & F: ATGAGTATTCTGATCACCCG & F: GCCTGGAGTTTTCCACTG & 666 \\
\hline & R: GAAGCGTTAGTGAGCCGTCTGCG & R: GACCAATAGCCGACAGCGTAG & \\
\hline \multirow[t]{2}{*}{ hisD } & F: GAAACGTTCCATTCCGCGC & F: GTCGGTCTGTATATTCCCGG & 894 \\
\hline & R: GCGGATTCCGGCGACCAG & R: GGTAATCGCATCCACCAAATC & \\
\hline \multirow[t]{2}{*}{ purE } & F: GACACCTCAAAAGCAGCGT & F: ACAGGAGTTTTAAGACGCATG & 510 \\
\hline & R: AGACGGCGATACCCAGCGG & R: GCAAACTTGCTTCATAGCG & \\
\hline \multirow[t]{2}{*}{ sucA } & F: CGCGCTCAAACAGACCTAC & F: CCGAAGAGAAACGCTGGATC & 643 \\
\hline & R: GACGTGGAAAATCGGCGCC & R: GGTTGTTGATAACGATACGTAC & \\
\hline \multirow[t]{2}{*}{ thrA } & F: GTCACGGTGATCGATCCGGT & F: ATCCCGGCCGATCACATGAT & 852 \\
\hline & R: CACGATATTGATATTAGCCCG & R: ACCGCCAGCGGCTCCAGCA & \\
\hline
\end{tabular}




\section{Clustered regularly interspaced short palindromic repeats (CRISPRs)}

11 strains of Salmonella Kentucky were further characterized by CRISPRs by two sets of specific primers. CRISPR1 locus was amplified with forward primer A1 (5'-ATAATGCTGCCGTTGGTAA-3') and reverse primer A2 (5'-TTGATGAGTATGGTGGTTGTGGT-3'). CRISPR2 locus was amplified using primer B1 (5'-CTG TATAAAAGCCTCCCC- $\left.3^{\prime}\right)$ and reverse primer B2 (5'GTTGGTAGAATGTGGTGC-3'). The PCR products were delivered to Sangon Biotech (Shanghai) Co., Ltd. for purification and sequencing. The sequencing results were uploaded to CRISPRfinder (https://crispr.i2bc. paris-saclay.fr/) to obtain the spacers of CRISPR1 and CRISPR2 (Grissa Vergnaud and Pourcel 2008). The directrepeat and spacer names were identified by PasteurCRISPR database for each spacer (http://www.pasteur.fr/ recherche/genopole/PF8/crispr/CRISPRDB.html) (Grissa Vergnaud and Pourcel 2007).

\section{Results}

\section{Salmonella prevalence and serotypes}

Of the total of 467 samples analyzed, 27 (5.8\%) were positive for Salmonella, of which the isolation rate from dogs was $7.08 \%(24 / 339)$ and the isolation rate from cats was only $2.31 \%(3 / 130)$. Table 2 shows the detailed prevalence of Salmonella in pet from pet hospitals and breeding base; 7 different serotypes were identified among the 27 positive Salmonella isolates. These serotypes were $S$. Kentucky, $S$. Typhimurium, $S$. Indiana, $S$. Derby, $S$. Sandiego, $S$. London, $S$. Rissen. The dominant serovar was $S$. Kentucky followed by $S$. Typhimurium, $S$. Indiana and $S$. Derby. At the same time, both $S$. Sandiego and $S$. London found only one sample from a dog, similarly $S$. Rissen found only one strain in a cat sample.

\section{Multilocus sequence typing (MLST) analysis}

Twenty-seven of the Salmonella isolates were amplified and sequenced using seven of housekeeping genes from 399 to $501 \mathrm{bp}$. Table 2 shows in detail the results of MLST analysis of Salmonella, eight different ST patterns were identified among these 27 Salmonella isolates. ST198 was the most prevalent STs in the study, represented by 11 (40.74\%), followed by ST17 (18.21\%), ST34 (11.11\%) and ST39 (11.11\%). Interestingly, ST17 and ST39 were detected simultaneously in cat and dog samples. At the same time, most of these Salmonella isolates came from the same pet hospital. Therefore, Salmonella has the risk of cross-infection between pets and can even cause zoonotic diseases. A minimum spanning tree of all STs from the different sources was generated using BioNumerics version 7.6 (Fig. 1).

\section{Clustered regularly interspaced short palindromic repeats (CRISPRs) analysis}

We used CRISPR to investigate the spacer sequences and direct repeat regions of 11 strains of Salmonella Kentucky (Table 3). Forty-eight different spacers were discovered, all spacers were 29-35 bp long. Among them, 32 bp (39/48) is dominant. CRISPR1 accounted for 29 and CRISPR2 accounted for 19. Eight new CRISPR spacers were found in CRISPR1 region and four new spacers were found in CRISPR2 loci respectively (Table 4). Figure 2 carefully shows the S.Kentucky different spacers arrangements between STs in CRISPR1 and CRISPR2. In CRISPR1, the number of spacers ranged from 10 to 16 ; for CRISPR2, the number of spacers ranged from 13 to 15. An interlinked dataset of different spacers revealed $9 \mathrm{KCTs}$ among the 11 isolates. Among them, S7, S14 from the same pet hospital were pointed out to belong to KCT2. This result indicates that Salmonella has a strong cross infection in the same area. On the other hand, KCT1, KCT2, КСТ3, КСТ5, and КCT7 are all from the same pet hospital. This result indicates that there is a phenomenon of microevolution in the CRISPR loci in the same serotype. The tested Salmonella Kentucky strains has a 29 bp identical highly conserved sequence $5^{\prime}$-CGG TTTATCCCCGCTGGCGCGGGGAACAC- $3^{\prime}$ in the conserved region in all CRISPR loci, consistent with the result published by Fabre (Fabre et al. 2012).

Table 2 Serotype distribution of 27 Salmonella isolates

\begin{tabular}{|c|c|c|c|c|}
\hline Strain no & O antigens & $\mathrm{H}$ antigens & Serotype & No. of isolates (\%) \\
\hline $\begin{array}{l}\mathrm{S} 1, \mathrm{~S} 5, \mathrm{~S} 6, \mathrm{~S} 7, \mathrm{~S} 8, \mathrm{~S} 9, \mathrm{~S} 14, \mathrm{~S} 15, \mathrm{~S} 16 \\
\mathrm{~S} 21, \mathrm{~S} 23\end{array}$ & 8,20 & $\mathrm{i}_{;} \mathrm{z}_{6}$ & S. Kentucky & $11(40.74 \%)$ \\
\hline $\mathrm{S} 11, \mathrm{~S} 12, \mathrm{~S} 13, \mathrm{~S} 18, \mathrm{~S} 19$ & $1,4,5,12$ & $\mathrm{i} ; 1,2$ & S.Typhimurium & $5(18.52 \%)$ \\
\hline S2, S3, S4, S22, T2 & $1,4,12$ & $z ; 1,7$ & S. Indiana & $5(18.52 \%)$ \\
\hline $\mathrm{S} 10, \mathrm{~S} 20, \mathrm{~T} 1$ & $1,4,12$ & $\mathrm{f}, \mathrm{g}$ & S. Derby & $3(11.11 \%)$ \\
\hline S17 & $1,4,5,12$ & $e, h ; e, n, z_{15}$ & S. Sandiego & $1(3.70 \%)$ \\
\hline S24 & 3 & $I, v ; 1,6$ & S. London & $1(3.70 \%)$ \\
\hline T3 & 6,7 & $\mathrm{f}, \mathrm{g}$ & S. Rissen & 1 (3.70\%) \\
\hline
\end{tabular}




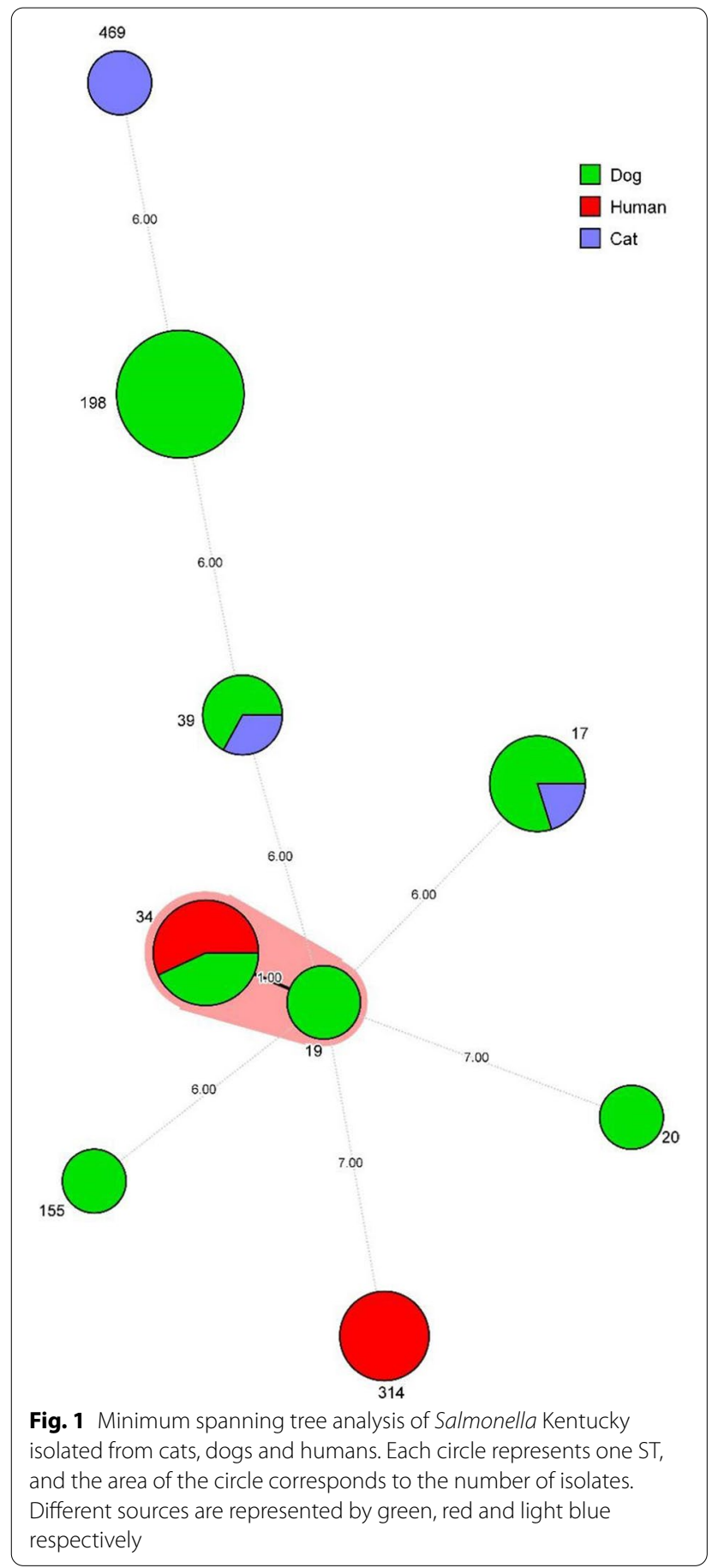

\section{Discussing}

In this study, Salmonella was isolated from pet dogs and cats in Xuzhou City, Jiangsu Province, China. The isolation rates were $7.08 \%$ and $2.31 \%$, respectively. The prevalence of Salmonella in dogs was similar in parts of Japan (Yukawa et al. 2019). However, the prevalence was lower than Ethiopia, Canada and Thailand (Kiflu
Table 3 Salmonella Kentucky strains used for molecular analysis in this study

\begin{tabular}{lll}
\hline Source & Strain (KCTs) & ST \\
\hline A & S6 (1), S7 (2), S8 (7), S14 (2), S15 (3), S9 & 198 \\
& $(5)$, S16 (6) & \\
B & S1 (8), S5 (4) & 198 \\
F & S21 (2) & 198 \\
G & S23 (9) & 198 \\
\hline
\end{tabular}

A, B, F, G: Different pet hospitals

KCTs: Kentucky CRISPR Types

et al. 2017; Leonard et al. 2011; Wu et al. 2020). On the other hand, the prevalence of Salmonella in pet cats was lower than that of dogs and humans, which was consistent with the findings of South Africa and Iraq (Zenadal-Obaidi and Al-Talibi 2014; GelawNthaba and Matle 2018). Worryingly, we found that Salmonella is more likely to infect older or young pets. This result is the same as the previous study (Reimschuessel et al. 2017; Jay-Russell et al. 2014; Tupler et al. 2012). In fact, some factors need to be considered when we analyze the prevalence of Salmonella in different regions, including pet hospital management, breeding condition, sampling method, sampling season and isolation method (Seepersadsingh Adesiyun and Seebaransingh 2004). In general, Salmonella will pose a great threat to human public health, especially for children and the elderly. For serotypes, the main widespread were Salmonella Kentucky and Salmonella Typhimurium in this study. Therefore, we analyze Salmonella with different molecular methods to determine its clonal structure and determining the diversity among STs in the same serovar.

Salmonella Kentucky has a wide range of prevalence and many hosts and through experiments, it has been found that compared with other serotypes, its drug resistance was generally stronger (Zankari et al. 2013). With the rapid development of molecular biology, the typing methods of Salmonella were gradually diversified. MLST and CRISPR molecular typing were commonly used typing methods in recent years. Combined with PCR technology, special bacterial gene fragments were amplified and sequenced. Through sequence comparison software, we can compare the differences of strains at the genetic level and understand the genetic relationship between strains. The resolution of the two methods were higher than that of traditional serotyping. MLST molecular typing relies on multiple conservative housekeeping gene sequences, with low sequence variation, good experimental reproducibility, and reliable results, which can distinguish the same serotypes 
Table 4 Salmonella Kentucky spacer name

\begin{tabular}{|c|c|c|}
\hline CRISPR locus & Spacer name ${ }^{a}$ (position) & Spacer DNA sequence $\left(5^{\prime}-3^{\prime}\right)$ \\
\hline 1 & Alt1 (1) & AAAAACTAAATCGCAAATAACCAAAAATATCA \\
\hline 1 & Conc15 (2) & TGGCGATAATGTACGACGAGTTGCCAAAATAT \\
\hline 1 & Ana3 (3) & ACCAAAACACTGAAAGCCATTCCTCTGGCGTA \\
\hline 1 & Ber1 (4) & TGACGGACAACGCGGCCAGTTAGTTTTACCTC \\
\hline 1 & Ken17 (5) & CGCAGCGAAGCGAGGACATAAAAGCATTACGG \\
\hline 1 & Ken18 (6) & CTGCGCCAGTGCGGTGATATCGTTGCTCATAG \\
\hline 1 & Ken19 (7) & GCCTACATAGCCGTAAAAGTCGTCCTGACTAA \\
\hline 1 & Ken20 (8) & TTAATACTGTCAACCAGCGCCCCCTGATTGTC \\
\hline 1 & Ken37 (9) & TTCGATATGAACGACGCGCAAGCAAAAGAATT \\
\hline 1 & Ken38 (10) & TTGCAGGCCCTGGTAGTTGACGCTATCGGGGC \\
\hline 1 & Ken39 (11) & GGCGTTCTGGTTCTGGCGGCTAAGATAAAGGG \\
\hline 1 & Newp8 (12) & GGGATCAGCACCGACAACCTAAAACCACTGTT \\
\hline 1 & Chin1var1 (13) & GTTATGAGTTTTGAGCGTTTTGTGCCGTCGCCC \\
\hline 1 & Ken $21^{*}(14)$ & GCAGCGGCGATACGTGTAAAAATCCCCTGGT \\
\hline 1 & Ken22* (15) & ATGTGACGATCTGCGGTGGTTACCAGCCAACA \\
\hline 1 & Ken40 (16) & GCAGCGGCGATACGTGGAAAAAACCCCCTGGT \\
\hline 1 & Ken41 (17) & AGGTGACGATCTGCGGTGGTTACCAGCCAACA \\
\hline 1 & Ken42 (18) & GTTATGAGTTTTGAGCGTTTTGTGCCGTCGCC \\
\hline 1 & Ken50 (19) & TGAGTCTCTATGGTTAGCAATAATGCGCACAT \\
\hline 1 & Ken51 (20) & CTCTCCAGTTCGGCCAGAAACTCCCTGGTCTG \\
\hline 1 & Ken52 (21) & GCGCTTTAACGCCCGCTCGCCCCTGAACCCCT \\
\hline 1 & Ken53 (22) & CTCCTCGCATTTGTGACTTTCTGGATCATCGG \\
\hline 1 & Ken40var1 (23) & GCAGCGGCGATACGTGGAAAAACCCCCCTGGT \\
\hline 1 & Ken23* (24) & GGGATCAACACCGACAAACCTAAAACCACTGTT \\
\hline 1 & Ken24* (25) & GCATCGGCGATATGTGGAAAAAACCCCCTGGTCG \\
\hline 1 & $\operatorname{Ken} 25^{*}(26)$ & AAAAACTAAATAAGCAAATAACCAAAAATATCA \\
\hline 1 & Ken26* $(27)$ & TGAGTCTCTATGGTTAGCAATAATGCGCACATC \\
\hline 1 & $\operatorname{Ken} 27^{*}(28)$ & GGCGTTCTGGTTCTGGCGGCTAAGAAAAGGCCG \\
\hline 1 & Ken28* (29) & GTGGATCATTACCGACATCCTAAAACCACTGTTTC \\
\hline 2 & EntB0 (1) & GGCTACACGCAAAAATTCCAGTCGTTGGCGCA \\
\hline 2 & MonB22 (2) & CCAGCCTCCCGTTACGCATATTTGAGGGTTTT \\
\hline 2 & MonB24 (3) & GCGGCGCTGATTATGATTTCCCGATAATTTAT \\
\hline 2 & $\mathrm{NiaB2}(4)$ & CTTTCGGGTTGAAAGCAGTAGCACTCATGCCC \\
\hline 2 & KenB29 (5) & CATTCCGGCGAGACTGAGCAGCGGGCGAACCG \\
\hline 2 & ParcB2 (6) & GGCCGTCTGCATTGAGCGCAGTTTTAACGCGT \\
\hline 2 & KenB30 (7) & TACGGATGTGTGACGGAGTCGCGTTTATGGCG \\
\hline 2 & KenB31 (8) & ACCGTTTCACGGATGAGAGTTGGCGGAAGCGC \\
\hline 2 & KenB32 (9) & CAGGAATAGACCTCTTTTTGCGCTGTGGCTTGC \\
\hline 2 & CholB4 (10) & TGCGCCACGCCTATACCATCCGAGTTTTGAGC \\
\hline 2 & BloB2 (11) & AACAAACTGGAGCGAAATTTCGCACATGCCCC \\
\hline 2 & KenB33 (12) & GTGCGGTTTCTTGCCGAATAGTGCCTCGTACT \\
\hline 2 & Tho6 (13) & TTCAGAGAATTGCCGCCAATAGAACAGCGCAA \\
\hline 2 & KenB42 (14) & AGTCTTTCAGTCCATTGGTCTATGATCTCTCG \\
\hline 2 & KenB43 (15) & ATGATGTATCGGCCATCGCGCCGGGTTATGTT \\
\hline 2 & KenB28* (16) & AACCAAACTGGAGCGAAATTTCGCACATGCCCCC \\
\hline 2 & KenB33* (17) & TTCAGAGAATTGCCGCCCATAGACAACCGCAA \\
\hline 2 & KenB34* (18) & ATGATGTATTCGGCATCGCGCCGGGTTATGTG \\
\hline 2 & KenB35* (19) & GGCTTCGTGCGCCGCACATCGCAGCGACT \\
\hline
\end{tabular}

${ }^{a}$ An asterisk shows a novel spacer identified in the current study 


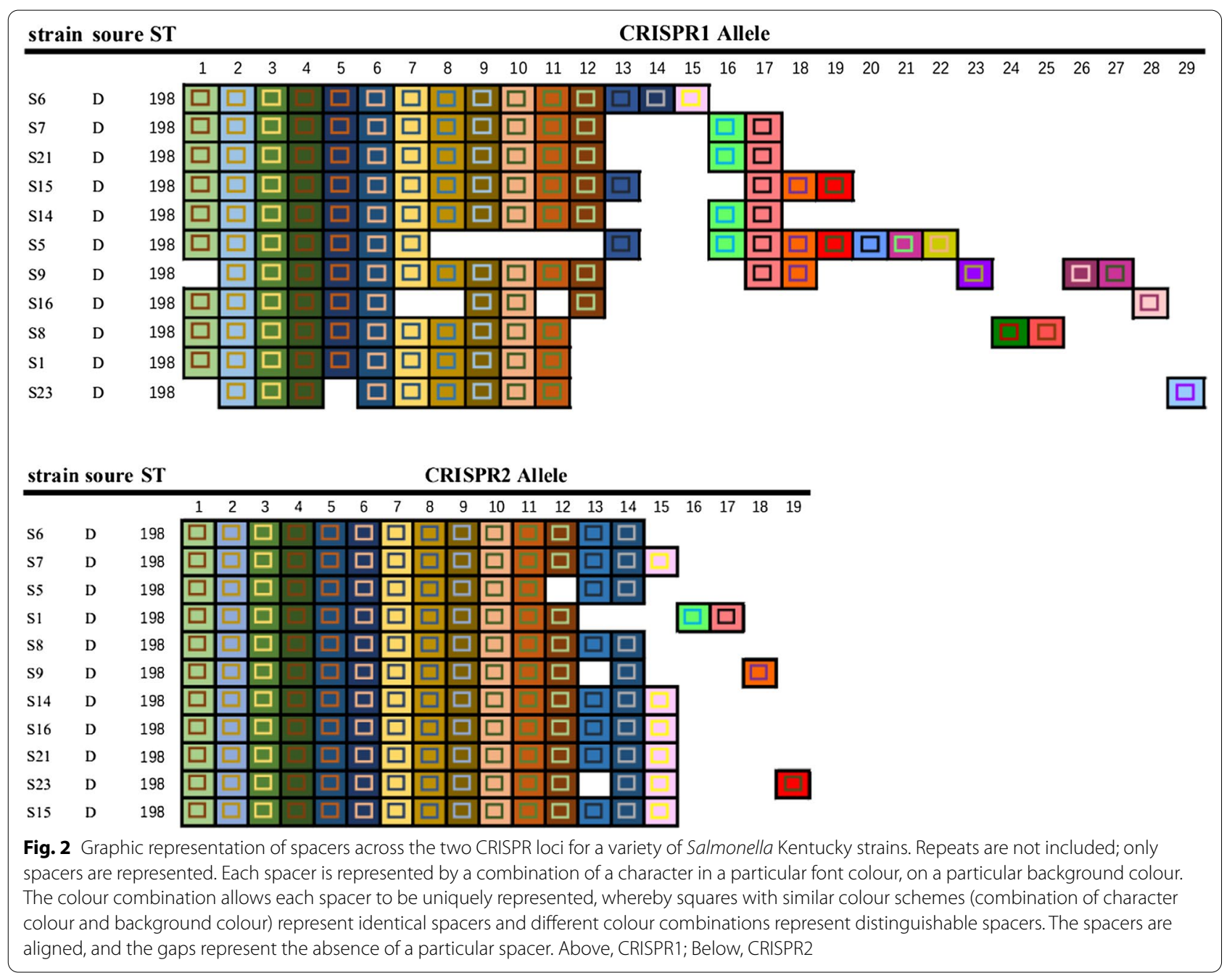

(Ho et al. 2017); while CRISPR molecular typing relies on the CRISPR loci of bacteria spacer sequence, the spacer sequence was a short DNA obtained from foreign nucleic acids, such as phage or plasmids, which were inserted into the bacterial chromosomes to prevent them from being infected by plasmids or homologous phages (Barrangou et al. 2007). Due to the diversity of phages and plasmids pools in the environment, different CRISPRs had emerged. Thus, CRISPRs had higher resolution in differentiate outbreak strains/ clones in epidemic clones (Liu et al. 2011). The deletion and insertion of the spacer sequence form a high degree of polymorphism of the sequence, which makes the CRISPR typing diversified. This method had high resolution to distinguish between strains with close relationships.

MLST was usually used to distinguish different Salmonella subtypes, and it can break through the limitations of traditional serotyping. We used MLST to perform rapid and accurate identification of serotyping of pet Salmonella from different sources. Each strain has been profiled by different seven alleles. The results showed that a total eight different STs were identified in 27 isolates, including ST198, ST17, ST34, ST19, ST39, ST20, ST469 and ST155. Among them, ST198 was the most frequent genotype recovered in this study. Salmonella Kentucky was found in many countries, such as the United States, Denmark, Britain, France and China, and its hosts mainly include poultry, pets and humans. Although both ST198 and ST314 in this study correspond to Salmonella Kentucky, but their seven pairs of housekeeping genes were not consistent. Le Hello et al. performed MLST typing on 66 isolates and obtained 9 ST types, ST198 was the dominant type, accounting for $74 \%$, ST314 was also detected, but the proportion was relatively low (Le Hello et al. 2011). Similarly, ST34 and ST19 are closely related. There was only one housekeeping gene $(d n a \mathrm{~N})$ between ST34 and ST19 that has been mutated. Previous 
experiments have shown that ST34 was more resistant than ST19 (Zankari et al. 2013), and both belong to Salmonella typhimurium. ST17 was the more common ST type of Salmonella from chickens in Jiangsu Province. In recent years, human infections caused by Salmonella Indiana have also increased (Gong et al. 2016). Finally, ST39, ST469, and ST155 appeared multiple times in animal-derived and food-borne Salmonella (Gong et al. 2016; Yang et al. 2016; Zhou et al. 2017). The ST type appearing in this study was widely present in all kinds of animals and animal products. It was very likely that pet dogs and cats have been in contact with other animals infected by Salmonella or eat contaminated food, and the infected pets may spread Salmonella to humans and cause outbreaks of salmonellosis through close contact with humans.

CRISPR typing was performed on 11 strains of Salmonella Kentucky, and the CRISPR1 and CRISPR2 loci of the strains were amplified and sequenced. By searching the CRISPRFinder database, we found the repeat sequences and spacer sequences, and draw sequence maps. The results showed that the fixed repeat sequences of the two loci were the same. There were 29 kinds of spacer sequences at the CRISPR1 loci, and 8 kinds of new spacer sequences have emerged; there were 19 kinds of spacer sequences at the CRISPR2 loci, and 4 new spacer sequences had appeared. Figure 2 showed that the spacers of CRISPR1 and CRISPR2 were diversity in S.Kentucky. The reason for this phenomenon was due to the duplication of a single spacer-DR units or the deletion of single or multiple spacer-DR units. The results of Fabre et al. showed that the number of CRISPR1 spacers in Salmonella Kentucky was 19 or 18, and the number of CRISPR2 spacers was 18 or 17 (Gong et al. 2016). In this article, most of the Salmonella Kentucky strains had repeat sequence deletions and insertions at both CRISPR loci, this phenomenon is called CRISPRS microevolution. In other words, the change process of the spacer loci was considered to be the evolution process of Salmonella, the dynamic changed of the spacer sequence in the locus can not only cause short-term phenotype changes, but also cause long-term subclass evolution (Fricke et al. 2011; Horvath et al. 2008). Salmonella Kentucky CRISPR typing (KCT) identified nine KCTs among the 27 isolates from pet dogs. This was consistent with the results of a previous study that CRISPR typing has a better ability to distinguish Salmonella Kentucky than MLST (Fen et al. 2011). It was found that multiple KCTs were separated in the same pet hospital. This diversity should be attributed to the sanitation and management conditions of the pet hospital.

According to the 11 strains of Salmonella Kentucky in this study were all ST198, CRISPR molecular typing divides the strains of the same ST type into multiple types. This proved that CRISPR typing is more detailed, not only had the ability to distinguish strains of different serotypes, but also had a certain ability to distinguish strains with high homology, with a higher resolution than MLST typing. However, due to the high variability of the bacterial CRISPR loci sequence and the continuous emergence of new spacer sequences, the CRISPR database had some shortcomings. The experimental data need to be continuously updated and improved by researchers. This was of great significance for data sharing in different countries and regions and for the epidemiological analysis of Salmonella.

\section{Acknowledgements}

This work was supported by the Graduate Student Research and Innovation Project of Jiangsu Normal University(2019XKT407, 2020XKT501, 2020XKT506), Priority Academic Program Development of Jiangsu Higher Education Institutions(PADD).

\section{Authors' contributions}

CY: Performed research and Wrote the paper. WS: Contributed new methods or models. LW: Analysis data. LC: Performed research. AZ: Conceived of or designed study. ZP: Conceived of or designed study. All authors read and approved the final manuscript.

\section{Funding}

The Graduate Student Research and Innovation Project of Jiangsu Normal University (2019XKT407): Cheng Yang. The Graduate Student Research and Innovation Project of Jiangsu Normal University (2020XKT501): Wangfeng Shao. The Graduate Student Research and Innovation Project of Jiangsu Normal University (2020XKT506): Lingxiao Chen. Priority Academic Program Development of Jiangsu Higher Education Institutions (PADD): Aihua Zhu.

Availability of data and materials

Please contact to the authors for all request.

\section{Declarations}

Ethics approval and consent to participate Not applicable.

\section{Consent for publication}

Not applicable.

\section{Competing interests}

The authors declare that there are conflicts of interest.

\section{Author details}

${ }^{1}$ College of Health Sciences, Jiangsu Normal University, Xuzhou 221116, Jiangsu, China. ${ }^{2}$ School of Life Sciences, Jiangsu Normal University, Xuzhou 221116, Jiangsu, China. ${ }^{3}$ Jiangsu Co-Innovationnovation Center for Prevention and Control of Important Animal Infectious Diseases and Zoonoses, Yangzhou University, Yangzhou 225009, Jiangsu, China. ${ }^{4}$ College of Arts and Sciences, Suqian University, Suqian 223800, Jiangsu, China.

Received: 24 December 2020 Accepted: 15 April 2021

Published online: 24 April 2021

\footnotetext{
References

Barrangou R, Fremaux C, Deveau H, Richards M, Boyaval P, Moineau S, Romero DA, Horvath P (2007) CRISPR provides acquired resistance against viruses in prokaryotes. Science 315:1709-1712
} 
Behravesh CB, Ferraro A, Deasy MR, Dato V, Moll M, Sandt C, Rea NK, Rickert R, Marriott C, Warren K, Urdaneta V, Salehi E, Villamil E, Ayers T, Hoekstra RM, Austin JL, Ostroff S, Williams IT (2010) Human Salmonella infections linked to contaminated dry dog and cat food, 2006-2008. Pediatrics 126:477-483

Cherry B, Burns A, Johnson GS, Pfeiffer H, Dumas N, Barrett D, McDonough $\mathrm{PL}$, Eidson M (2004) Salmonella typhimurium outbreak associated with veterinary clinic. Emerg Infect Dis 10:2249-2251

Fabre L, Zhang J, Guigon G, Le Hello S, Guibert V, Accou-Demartin M, de Romans S, Lim C, Roux C, Passet V, Diancourt L, Guibourdenche M, Issenhuth-Jeanjean S, Achtman M, Brisse S, Sola C, Weill FX (2012) CRISPR typing and subtyping for improved laboratory surveillance of Salmonella infections. PLOS ONE 7:e36995

Fen L, Kariyawasam S, Jayarao BM, Barrangou R, Gerner-Smidt P, Ribot EM, Knabel SJ, Dudley EG (2011) Subtyping Salmonella enterica serovar enteritidis isolates from different sources by using sequence typing based on virulence genes and clustered regularly interspaced short palindromic repeats (CRISPRs). Appl Environ Microbiol 77:4520-4526

Fricke WF, Mammel MK, McDermott PF, Tartera C, White DG, LeClerc JE, Ravel J, Cebula TA (2011) Comparative genomics of 28 Salmonella enterica isolates: evidence for CRISPR-mediated adaptive sublineage evolution. $J$ Bacteriol 193:3556-3568

Gelaw AK, Nthaba P, Matle I (2018) Detection of Salmonella from animal sources in South Africa between 2007 and 2014. J South Afr Vet Assoc $1-10$.

Gong J, Wang C, Shi S, Bao H, Zhu C, Kelly P, Zhuang L, Lu G, Dou X, Wang R, Xu Bu, Zou J, Shi H (2016) Highly drug-resistant Salmonella enterica serovar indiana clinical isolates recovered from broilers and poultry workers with diarrhea in China. Antimicrob Agents Chemother 60:1943-1947

Grissa I, Vergnaud G, Pourcel C (2007) The CRISPRdb database and tools to display CRISPRs and to generate dictionaries of spacers and repeats. BMC Bioinformatics 8:172-172

Grissa I, Vergnaud G, Pourcel C (2008) CRISPRcompar: a website to compare clustered regularly interspaced short palindromic repeats. Nucleic Acids Res 36:W145-W148

Gymoese P, Sørensen G, Litrup E, Olsen JE, Nielsen EM, Torpdahl M (2017) Investigation of outbreaks of Salmonella enterica serovar typhimurium and its monophasic variants using whole-genome sequencing, Denmark. Emerg Infect Dis 23:1631-1639

Ho Y, Chou M, Tsai H, Huang T, Fan C, Hsu B (2017) Empirical testing of modified Salmonella MLST in aquatic environmental samples by in silico analysis. Sci Total Environ 581-582:378-385

Horvath P, Romero DA, Couté-Monvoisin A, Richards M, Deveau H, Moineau S, Boyaval P, Fremaux C, Barrangou R (2008) Diversity, activity, and evolution of CRISPR Loci in Streptococcus thermophilus. J Bacteriol 190:1401-1412

Jay-Russell MT, Hake AF, Bengson Y, Thiptara A, Nguyen T (2014) Prevalence and characterization of Escherichia coli and Salmonella Strains isolated from stray dog and coyote feces in a major leafy greens production region at the United States-Mexico Border. PLoS ONE 9:1-14

Kidgell C, Reichard U, Wain J, Linz B, Torpdahl M, Dougan G, Achtman M (2002) Salmonella typhi, the causative agent of typhoid fever, is approximately 50,000 years old. Infect Genet Evol 2:39-45

Kiflu B, Alemayehu H, Abdurahaman M, Negash Y, Eguale T (2017) Salmonella serotypes and their antimicrobial susceptibility in apparently healthy dogs in Addis Ababa, Ethiopia. BMC Vet Res 13:1-9

Koehler KM, Lasky T, Fein SB, DeLong SM, Hawkins MA, Rabatsky-Ehr T, Ray SM, Shiferaw B, Swanson E, Vugia DJ (2006) Population-based incidence of infection with selected bacterial enteric pathogens in children younger than five years of age, 1996-1998. Pediatr Infect Dis J 25:129-134

Le Hello S, Hendriksen RS, Doublet B, Fisher I, Nielsen EM, Whichard JM, Bouchrif B, Fashae K, Granier SA, Jourdan-Da Silva N, Cloeckaert A, Threlfall EJ, Angulo FJ, Aarestrup FM, Wain J, Weill FX (2011) International spread of an epidemic population of Salmonella enterica serotype kentucky ST198 resistant to ciprofloxacin. J Infect Dis 204:675-684

Leonard EK, Pearl DL, Finley RL, Janecko N, Peregrine AS, Reid-Smith RJ, Weese JS (2011) Evaluation of pet-related management factors and the risk of Salmonella spp. carriage in pet dogs from volunteer households in Ontario (2005-2006). Zoonoses Public Health 58:140-149
Murphy C, Reid-Smith RJ, Prescott JF, Bonnett BN, Poppe C, Boerlin P, Weese JS, Janecko N, McEwen SA (2009) Occurrence of antimicrobial resistant bacteria in healthy dogs and cats presented to private veterinary hospitals in southern Ontario: a preliminary study. Can Vet J 50:1047-1053

Reimschuessel R, Grabenstein M, Guag J, Nemser SM, Song K, Qiu J, Clothier KA, Byrne BA, Marks SL, Cadmus K, Pabilonia K (2017) Multilaboratory survey to evaluate Salmonella Prevalence in diarrheic and nondiarrheic dogs and cats in the multilaboratory survey to evaluate Salmonella prevalence in diarrheic and nondiarrheic dogs and cats in theUnited States between 2012 and 2014. J Clin Microbiol 55:1350-1360

Reimschuessel R, Grabenstein M, Guag J, Nemser SM, Song K, Qiu J, Clothier KA, Byrne BA, Marks SL, Cadmus K, Pabilonia K, Sanchez S, Rajeev S, Ensley S, Frana TS, Jergens AE, Chappell KH, Thakur S, Byrum B, Cui J, Zhang Y, Erdman MM, Rankin SC, Daly R, Das S, Ruesch L, Lawhon SD, Zhang S, Baszler T, Diaz-Campos D, Hartmann F, Okwumabuaq O (2017) Multilaboratory survey to evaluate Salmonella prevalence in diarrheic and nondiarrheic dogs and cats in the United States between 2012 and 2014. J Clin Microbiol 55:1350-1368

Seepersadsingh N, Adesiyun AA, Seebaransingh R (2004) Prevalence and antimicrobial resistance of Salmonella spp. in non-diarrhoeic dogs in Trinidad. J Vet Med Ser B 51:337-342

Shi C, Singh P, Ranieri ML, Wiedmann M, Moreno Switt Al (2013) Molecular methods for serovar determination of Salmonella. Crit Rev Microbiol 41:309-325

Tennant SM, Diallo S, Levy H, Livio S, Sow SO, Tapia M, Fields PI, Mikoleit M, Tamboura B, Kotloff KL, Nataro JP, Galen JE, Levine MM (2010) Identification by PCR of non-typhoidal Salmonella enterica serovars associated with invasive infections among febrile patients in Mali. PLoS Negl Trop Dis 4:e621

Thompson CP, Doak AN, Amirani N, Schroeder EA, Wright J, Kariyawasam S, Lamendella R, Shariat NW (2018) High-resolution identification of multiple Salmonella serovars in a single sample by using CRISPR-SeroSeq. Appl Environ Microbiol. https://doi.org/10.1128/AEM.01859-18

Tupler T, Levy JK, Sabshin SJ, Tucker SJ, Greine EC, Leutenegger CM (2012) Enteropathogens identified in dogs entering a Florida animal shelter with normal feces or diarrhea. Sci Rep 241:338-343

Van Immerseel F, Pasmans F, De Buck J, Rychlik I, Hradecka H, Collard JM, Wildemauwe C, Heyndrickx M, Ducatelle R, Haesebrouck F (2004) Cats as a risk for transmission of antimicrobial drug-resistant Salmonella. Emerg Infect Dis 10:2169-2174

Wray C, Wray A (2000) Salmonella in domestic animals. J S Afr Vet Assoc $71: 104-143$

Wu X, Angkititrakul S, Richards AL, Pulsrikarn C, Khaengair S, Keosengthong A, Siriwong S, Suksawat F (2020) Risk of antimicrobial resistant nontyphoidal Salmonella during asymptomatic infection passage between pet dogs and their human caregivers in Khon Kaen, Thailand. Antibiotics 9:477

Yang X, Huang J, Wu Q, Zhang J, Liu S, Guo W, Cai S, Yu S (2016) Prevalence, antimicrobial resistance and genetic diversity of Salmonella isolated from retail ready-to-eat foods in China. Food Control 60:50-56

Yukawa S, Uchida I, Tamura Y, Ohshima S, Hasegawa T (2019) Characterisation of antibiotic resistance ofSalmonellaisolated from dog treats in Japan. Epidemiol Infect 147:e102

Zankari E, Hasman H, Kaas RS, Seyfarth AM, Agerso Y, Lund O, Larsen MV, Aarestrup FM (2013) Genotyping using whole-genome sequencing is a realistic alternative to surveillance based on phenotypic antimicrobial susceptibility testing. J Antimicrob Chemother 68:771-777

Zenad MM, Al-Obaidi QT, M. A. AL-Talibi, (2014) Prevalence of Salmonella species in stray cats in mosil city, Irao. Online J Anim Feed Res 5:133-136

Zhou Z, Li J, Zheng H, Jin X, Shen Y, Lei T, Sun X, Pan Z, Jiao X (2017) Diversity of Salmonella isolates and their distribution in a pig slaughterhouse in Huaian, China. Food Control 78:238-246

\section{Publisher's Note}

Springer Nature remains neutral with regard to jurisdictional claims in published maps and institutional affiliations. 